\section{Doing Drugs}

It's not difficult to find online databases containing pharmaceutical info. A little Googling leads easily to molecular structures, lab tests, patents, side effects, and much miscellany. What's not so easy to find is a database that links drugs with data and the proteins being targeted. Fortunately, that important niche is occupied by David Wishart's DrugBank. The list of compounds in the database is impressive-over 3000 experimental compounds, greater than 1000 FDA-approved small molecules, 113 FDA-approved protein/peptides, $60+$ nutraceuticals, and over 6000 protein targets (complete with sequences). Wide-

\section{Proper IDs}

You're out in the field, and you spot a really interesting looking tropical plant. It's not one you're familiar with, and the printed field guide you own doesn't list it. What to do? To the rescue comes the Virtual Field Herbarium (VFH) complete with its gallery of thousands of images of plants. The

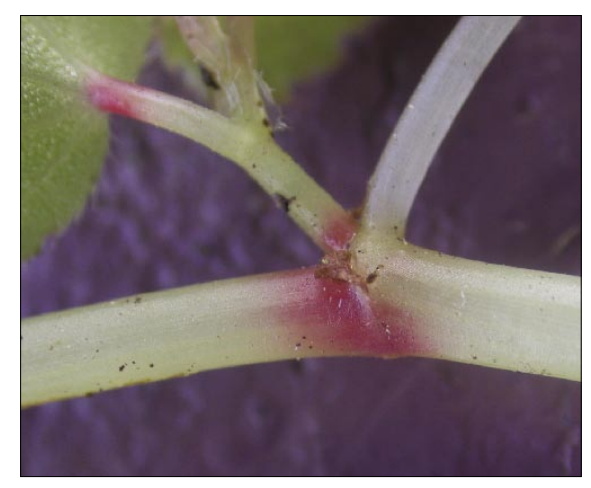

subjects of most photos in the collection are available as herbarium specimens, and the original photographer or collector of each is known, so questionable identifications can be readily rechecked. Recognizing that no single field guide can be everything to everyone, VFH goes way beyond the standard reference, with tips on designing personalized field guides and then to "make it happen," provides an extensive set of links/listings to existing field guides and relevant information. Its bibliography alone, with over 800 entries, is a bonanza for tropical botanists.

\section{herbaria.plants.ox.ac.uk/vfh}

\section{Big Field Microscope}

With content areas that span news, images, education, historical documents, taxonomic indexing of organisms, and others, it's not much of an exaggeration to describe Micro*scope as the mother of all microscopy sites on the web. Designed with a relaxed taxonomic approach to managing organism information, the site is loosely hierarchical in its organization so as to allow visitors the maximum likelihood of easily finding desired info. Collections can be browsed alphabetically, by topic, or by concept. Organisms can similarly be viewed alphabetically or by classification. Micro*scope appears to be meeting with great success, if the recent addition of its 10,000th image is any indication.

\section{starcentral.mbl.edu/microscope/ portal.php}

\section{Cellular Communication}

Supported by the National Institute of General Medical Sciences and the National Institute of Allergy and Infectious Diseases, the Alliance for Cell Signaling (AfCS) has some heavy hitters behind it and with good reason-the group's goals are to "understand as completely as possible the relationships between sets of inputs and outputs in signaling cells that vary both temporally and spatially." In a word, it's all about communicationboth cellular and scientific. For scientists, there are full-length research reports and mini-communications about signaling that are freely available to visitors. With respect to cellular messaging, databases are available on very diverse signaling-related topics, including yeast two-hybrid analyses, antibodies, plasmids, phosphoproteins, and covalent modification. A signaling gateway puts frosting on the cake with up-to-the-minute news on this important topic.

\section{www.afcs.org}

\section{Busy Highways}

In the middle of Los Angeles, surrounded by a bewildering array of freeways pointed in all directions, you wouldn't dream of getting around without a map, so shouldn't scientists hoping to navigate the "interstate" pathways of cellular information also have some assistance? Enter Pathguide, the pathway resource list, which serves as a sort of super Rand McNally for scientists traveling across intracellular molecular freeways. With links to over 220 molecular "highways," Pathguide guides users to databases covering protein-protein interactions, metabolic pathways, signaling pathways, pathway diagrams, regulatory networks, protein-compound interactions, genetic interaction networks, and sequences of interest.

\section{www.pathguide.org/index.php}

- Kevin Ahern - Please send web site recommendations to ahernk@orst.edu 